\title{
La evaluación de las prácticas profesionales de coenseñanza
}

Carlos Arriagada Hernández ${ }^{10}$, Natalie Gislaine Venegas Pérez ${ }^{(10}$, Óscar Ovidio Calzadilla-Pérez ${ }^{(1)}$ Universidad Autónoma de Chile, Fundación del Magisterio de La Araucanía, Universidad Católica Temuco, Chile

Autor de correspondencia: ${ }^{1}$ carlos.arriagada@uautonoma.cl Recibido: 25 de marzo de 2020 Revisado: 20 mayo de 2020 Aprobado: 22 de septiembre de 2020 Publicado: 25 de febrero de 2021

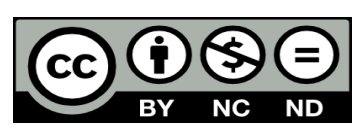

\section{Resumen}

La coenseñanza es una estrategia reconocida internacionalmente por sus potencialidades en el desarrollo profesional docente y en el aprendizaje de los estudiantes, que implica considerables transformaciones en el aula escolar. El Ministerio de Educación chileno implementó esta estrategia mediante los Programas de Integración Escolar, al señalarla como una herramienta para la mejora de la calidad de la educación. En consecuencia, este trabajo centra su objetivo en la evaluación de las prácticas de coenseñanza por medio de un estudio descriptivo y mixto que se apoya en la entrevista y el grupo focal. Este último se convirtió en un escenario de comprensión y reflexión sobre la gestión pedagógica y curricular de los docentes a propósito de las prácticas de coenseñanza. A partir de esta evaluación, se identificaron tanto dificultades en el área de gestión del tiempo para la preparación de la enseñanza como la prevalencia del trabajo aislado por parte del docente de aula y del educador diferencial. Las acciones de mejora instrumentada fortalecieron el liderazgo pedagógico, el diseño de la enseñanza, las relaciones interpersonales, el desarrollo profesional docente y el aprendizaje de los estudiantes.

Palabras clave: coenseñanza, liderazgo, formación profesional, trabajo estudiantil 


\title{
Evaluation of professional co-teaching practices
}

\begin{abstract}
Co-teaching is an internationally recognized strategy for its possibilities on teacher professional development and student learning, which involves considerable transformations in the classroom. The Chilean Ministry of Education implemented this strategy through the School Integration Programs, pointing to it as a tool for improving the quality of education. Consequently, this article focuses on the evaluation of co-teaching practices through a descriptive and mixed study based on the interview and the focus group. The latter became an occasion for understanding and reflecting on teachers' pedagogical and curricular management concerning co-teaching practices. From this evaluation, we identified both difficulties regarding time management for teaching preparation and the prevalence of isolated work on the part of the classroom teacher and the differential educator. The instrumented improvement actions strengthened pedagogical leadership, teaching design, interpersonal relations, teacher professional development and student learning.
\end{abstract}

Keywords: co-teaching, leadership, professional training, student work

\section{Avaliação das práticas profissionais de coensino}

\section{Resumo}

O coensino é uma estratégia reconhecida internacionalmente por seu potencial no desenvolvimento profissional dos professores e na aprendizagem dos alunos, o que envolve transformações consideráveis na sala de aula. O Ministério da Educação chileno implementou essa estratégia através dos Programas de Integração Escolar, destacando-a como uma ferramenta para melhorar a qualidade da educação. Assim, este artigo focaliza a avaliação das práticas de coensino através de um estudo descritivo e misto que se baseia na entrevista e no grupo focal. Este último tornou-se um espaço de compreensão e reflexão sobre a gestão pedagógica e curricular dos professores a respeito das práticas de coensino. A partir desta avaliação, foram identificadas dificuldades na área de gestão do tempo para a preparação do ensino, bem como a prevalência de trabalho isolado por parte do professor em sala de aula e do educador diferencial. As ações de melhoria implementadas fortaleceram a liderança pedagógica, o desenho do ensino, as relações interpessoais, o desenvolvimento profissional dos professores e a aprendizagem dos alunos.

Palavras-chave: coensino, liderança, formação profissional, trabalho estudantil

En el discurso actual de académicos, directivos y docentes, se percibe la significación y perentoriedad de originar y priorizar el respeto de la inclusión de los derechos de las personas, con o sin necesidades educativas especiales [NEE]. Esto se pone de manifiesto en la construcción de políticas públicas de algunos Estados y en la gestión de organizaciones 
internacionales ${ }^{1}$, las cuales en los últimos años, abordan entre los temas más recurrentes de sus agendas la inclusión educativa y las estrategias para su concreción (OCDE, 2004; ONU, 1993; Unesco, 1994, 2000).

Lo anterior también se refleja en el sistema educativo chileno, ya que el Ministerio de Educación [MinEduc] realiza esfuerzos para responder a las demandas de sujetos con NEE. Así, institucionalizó en su estructura ministerial la Unidad de Educación Especial mediante el Decreto 490/1990 (MinEduc, 1990). Esta unidad se ha encargado de generar políticas públicas y conocimientos especializados - servicios y recursos de apoyo- en congruencia con la definición de educación especial o diferencial contenida en la Ley General de Educación (MinEduc, 2009a).

Las estadísticas ofrecidas por el MinEduc avalan la necesidad de la búsqueda sistemática de estrategias efectivas para la atención integral de los 416000 estudiantes con NEE. 294000 corresponden a NEE transitorias y los restantes 122000 a permanentes. Ellos son atendidos en 40000 escuelas especiales y 82000 establecimientos educacionales con equipos del Programa de Integración Escolar [PIE] (MinEduc, 2019).

Una de las estrategias del MinEduc para responder a la atención de niños, adolescentes y jóvenes con NEE es la implementación de los equipos del PIE (MinEduc, 1990). Estos tienen entre sus objetivos favorecer la presencia, participación y logro de los aprendizajes de los estudiantes, especialmente de aquellos que presenten NEE. Los equipos del PIE se rigen por el Decreto 170/2009 (MinEduc, 2009b), el cual norma el diagnóstico de los estudiantes con NEE que son beneficiados por la Subvención de Educación Especial, y permite la contratación de diversos profesionales para brindar los apoyos según las necesidades de los estudiantes y del establecimiento.

De dicho decreto se desprenden las Orientaciones Técnicas para los PIE, donde se plantea como estrategia el trabajo colaborativo de docentes de aulas regulares y educadores diferenciales (MinEduc, 2013b). A través de los equipos de profesionales de los PIE, se ponen a disposición recursos humanos y materiales adicionales para proporcionar ayudas y apoyos y equiparar oportunidades de aprendizaje y participación. Los PIE se desarrollan en un establecimiento educacional o en grupos de establecimientos, como es el caso de los comunales y los ubicados en escuelas rurales multigrado. En consideración con la estructura de enseñanza del sistema escolar regular, y de acuerdo con la Ley General de Educación (MinEduc, 2009a), los PIE atienden estudiantes desde los niveles de transición I y II de la educación parvularia, las educaciones básica y media, hasta llegar a la educación de adultos (MinEduc, 2013a, 2013b, 2016).

En este sentido, en las Orientaciones Técnicas para los PIE (MinEduc, 2013b), se concibe el trabajo colaborativo en términos de "una de las principales herramientas para mejorar la calidad de los aprendizajes de todos los estudiantes, en particular de los que presentan NEE" (p. 39). En este contexto de análisis, las denominaciones del trabajo colaborativo y sus variantes en la educación especial tienen como núcleo común, por parte de sus profesionales, la búsqueda de conocimientos y estrategias de ayuda y apoyos para la atención de las NEE

1 Entre las convenciones y declaraciones se pueden destacar: la Conferencia Mundial sobre Educación para Todos (Unesco, 1990); la Declaración de Salamanca y Marco de Acción (Unesco, 1994); las Normas Uniformes para la Equiparación de Oportunidades para Personas con Discapacidad (ONU, 1993); la Convención Interamericana para la Eliminación de Todas las Formas de Discriminación contra las Personas con Discapacidad, entre otras. 
(González, 2010; Guirado et al., 2017; Iglesias, 2018; Molina, 2015; Moran et al., 2017; Soto, 2016). Como consecuencia, hay bibliografía especializada de términos, tales como: coenseñanza, co-docencia, enseñanza colaborativa, enseñanza en equipo o co-teaching. En general, estas se definen como la responsabilidad profesional que comparten dos o más personas en la educación de los estudiantes de forma colaborativa (Urbina et al., 2017; Villa et al., 2008).

En lo particular, la estrategia de coenseñanza compromete la acción de dos o más profesionales en tareas de planificación, instrucción y evaluación de un curso, que comparten la responsabilidad de enseñar a algunos o a todos los alumnos asignados a un aula escolar (MinEduc, 2013b; Murawski, 2008; Rodríguez, 2014). De hecho, la coenseñanza se enmarca en un conjunto de agrupaciones conceptuales respecto a la colaboración entre profesionales (Suárez, 2016; Vance, 2001). Por otra parte, las publicaciones de Friend et al. (2010) y Villa et al. (2008) mencionan criterios respecto a la taxonomía de las prácticas de coenseñanza.

Las publicaciones consultadas ponderan el valor socio-psico-pedagógico de la coenseñanza, pues contribuye a: la interacción entre los coenseñantes, la interdependencia positiva para atender la diversidad y el desarrollo de habilidades interpersonales (MinEduc, 2013b), así como la integración multi- e interdisciplinaria entre profesionales, la combinación de competencias curriculares en función de una meta para todos los alumnos y el intercambio de estrategias didácticas (Marsiglia-Fuentes et. al., 2020; Rodríguez, 2014). Estas potencialidades sirven para argumentar la prioridad que la Unidad de Educación Especial del MinEduc le confiere a la coenseñanza para el despliegue de los equipos del PIE.

Lo planteado contradice los resultados de la investigación desarrollada por la Fundación Chile (2013), que señala que los equipos del PIE se han caracterizado por "una débil implementación del trabajo colaborativo" (p. 256). Entre las causas que generan esta problemática se argumenta la escasa formación inicial en competencias de trabajo en equipo, el desconocimiento de los beneficios de la estrategia de coenseñanza, la sobrecarga administrativa y las dificultades a nivel de cohesión y organización escolar.

Lo antes expuesto cimentó la conjetura científica para ejecutar una investigación en la Escuela El Tesoro, ubicada en el sector rural San Ramón de la comuna de Padre Las Casas, IX región de La Araucanía, Chile. En consecuencia, el presente artículo tiene como objetivo: analizar el rol de la evaluación de las estrategias de coenseñanza empleadas por docentes de aula común, educadores diferenciales y psicopedagogos, mediante la identificación de fortalezas y necesidades de mejora que conduzcan al perfeccionamiento de la gestión de los equipos del PIE y al desarrollo profesional docente.

\section{Metodología}

La presente investigación se orienta hacia la evaluación de las prácticas profesionales de aplicación de la coenseñanza mediante la comprensión de las percepciones y comportamientos contextualizados de docentes de aula y educadores diferenciales, como fuente para orientar la toma de decisiones a nivel de establecimiento educativo, y, en consecuencia, para la mejora de las prácticas (Arriagada \& Calzadilla-Pérez, 2018). Esta investigación educativa posee un enfoque descriptivo - es decir "mide, evalúa o recolecta datos sobre diversos conceptos (variables), aspectos, dimensiones o componentes del 
fenómeno a investigar" (Hernández et al., 2006, p. 102) — y recoge antecedentes cualitativos y cuantitativos. En general, se comparan resultados a partir de la aplicación de entrevistas aplicadas en dos grupos focales, así como las transformaciones en la percepción de los docentes y la comprensión de las estrategias de coenseñanza. Esta última se representó mediante el empleo del software Atlas.ti.

\section{Participantes}

Los participantes de la investigación son docentes que implementan la estrategia de coenseñanza desde el nivel de transición I de la educación parvularia hasta octavo año de la educación básica en la escuela El Tesoro. En general, participan ocho docentes de educación general básica y tres profesoras de educación diferencial, que conforman una muestra no probabilística. El criterio de selección consistió en la determinación de los docentes responsables de ejecutar en el establecimiento la estrategia de coenseñanza.

\section{Instrumentos}

De acuerdo con la problemática planteada, y en respuesta a la limitación de instrumentos de evaluación y de orientaciones para la mejora de la estrategia de coenseñanza, se utilizaron dos procedimientos de evaluación. En primera instancia, una entrevista por medio de grupos focales que, según Mella (2010), "son ante todo una técnica de investigación cualitativa, donde la discusión grupal se utiliza como un medio para generar entendimiento profundo de las experiencias y creencias de los participantes" (p. 23). La discusión se enmarcó en preguntas relacionadas con la preparación, ejecución y reflexión a partir de la coenseñanza, por lo que se adaptaron las preguntas orientadoras de Villa et al. (2008).

Los datos cuantitativos de la investigación se obtuvieron de la adaptación del instrumento de autoevaluación Are we really co-teachers? (Villa et al., 2008), que se presenta en formato de encuesta y en el que las dimensiones se relacionan con la cultura de colaboración a propósito de las percepciones de los coenseñantes acerca de esta estrategia, tal como se muestra en la figura 1.

\section{Figura 1}

Dimensiones constituyentes del estudio de evaluación de las estrategias de coenseñanza

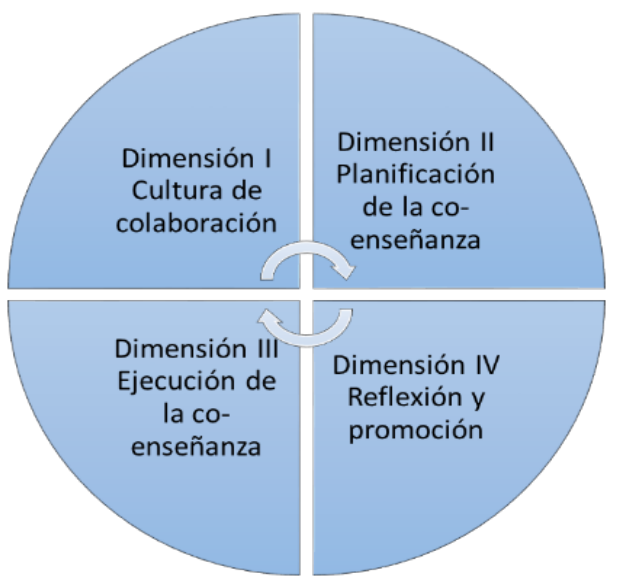




\section{Estrategia de investigación}

A continuación, se presenta el procedimiento investigativo empleado para la evaluación de la efectividad de la aplicación de la estrategia de coenseñanza:

- Análisis, revisión de dimensiones y traducción de las preguntas orientadoras del instrumento Are we really co-teachers? de Villa et al. (2008).

- Adaptación lingüística y de contenido, según orientaciones del MinEduc para la implementación de la coenseñanza.

- Valoración del instrumento - por al menos tres jueces expertos-.

- Desarrollo de grupos focales para conocer percepciones de la coenseñanza.

- Aplicación del instrumento de autoevaluación.

- Análisis de los datos obtenidos en el grupo focal para sacar conclusiones y elaborar el informe final.

- Análisis de resultados cuantitativos por medio de Excel y producción de gráficos del instrumento de autoevaluación.

- Agrupación de percepciones en gráficos elaborados en el software Atlas.ti y comparación de los resultados.

- Presentación de resultados a los docentes del establecimiento e identificación de fortalezas y necesidades de mejora en conjunto con docentes coenseñantes, según los resultados de la evaluación.

- Adaptación de A guide to co-teaching: New lessons and strategies to facilitate student learning (3ra. ed.) (Villa et al., 2008).

- Aplicación de la guía de coenseñanza.

- Valoración de los resultados a partir de las estrategias desarrolladas.

\section{Resultados}

La evaluación de la percepción y la comprensión de la coenseñanza por parte de los docentes se realiza por medio de la aplicación y el análisis de resultados del instrumento Are we really co-teachers? (Villa et al., 2008).

Los resultados relativos a la dimensión I —cultura de colaboración- (figura 2) indican que los participantes declaran que tienen ideas comunes respecto al proceso de enseñanza. El 90 \% de los encuestados valora como positiva la aplicación de acciones de coenseñanza. El $60 \%$ de profesores reconoce la coenseñanza como uno de los factores claves para desarrollar una clase efectiva, y un 40 \% señala la modelación, la colaboración y el trabajo en equipo, además de la solución de conflictos.

En relación con la dimensión II — planificación de la coenseñanza- (figura 3), la mitad de los participantes señala que frecuentemente realizan en conjunto las adecuaciones curriculares según las NEE de los estudiantes. Asimismo, los encuestados consideran que se reúnen frecuentemente junto al codocente para acciones de planificación de la enseñanza, donde se definen los roles, enfoques de trabajo colaborativo y se comparten responsabilidades 
en la preparación de la enseñanza. Por otra parte, un $20 \%$ de los participantes afirma que antes de las clases definen los roles y las funciones de cada coenseñante. Así mismo sucede con la determinación conjunta de los procedimientos de monitoreo y evaluación de los aprendizajes.

\section{Figura 2}

Dimensión I: cultura de colaboración

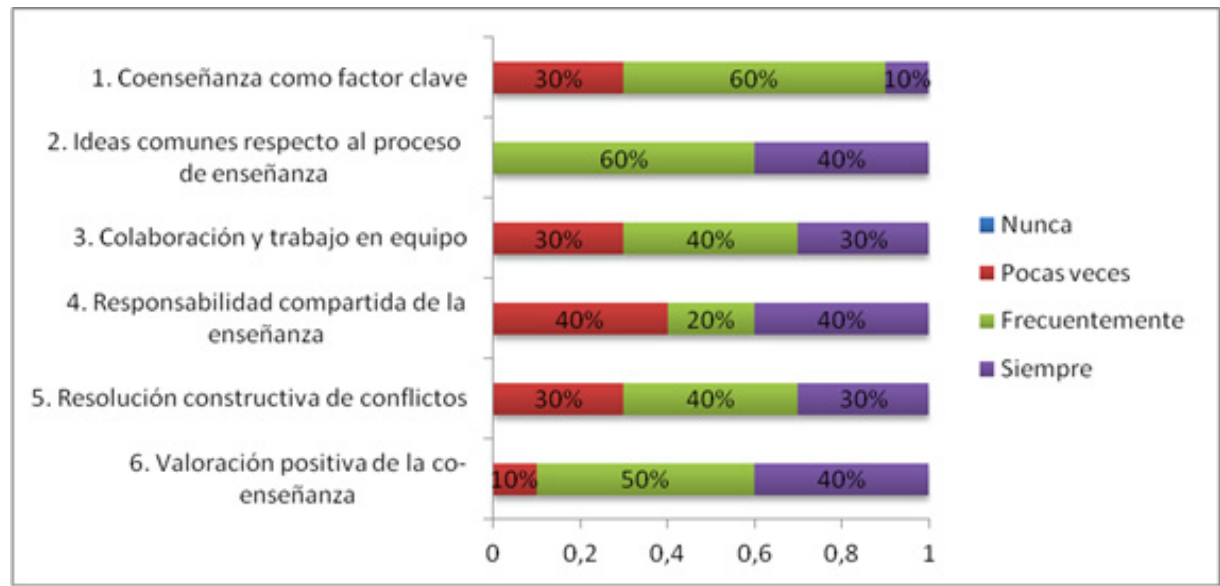

Figura 3

Dimensión II: planificación de la coenseñanza

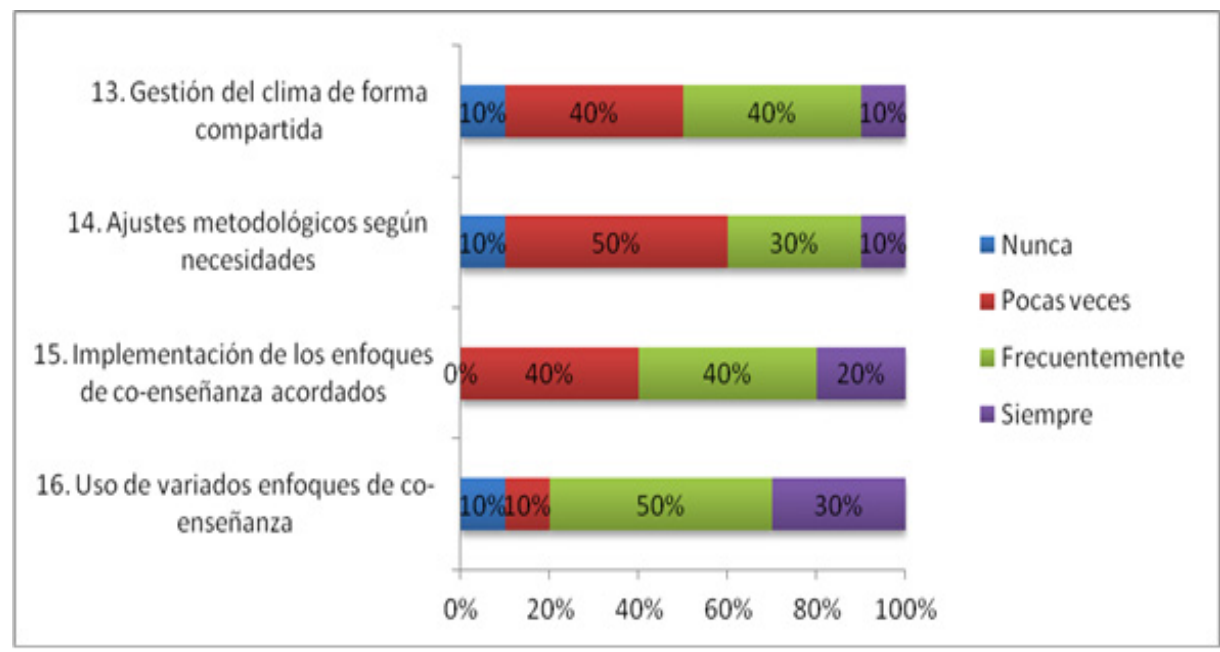

Enla dimensión III - ejecución de la coenseñanza-(figura 4), la mitad de los participantes señala que frecuentemente utilizan variados enfoques de enseñanza compartida en el aula. Existe una tendencia positiva, en un $60 \%$, sobre la implementación efectiva del enfoque acordado en el momento de la planificación. Por otra parte, la mitad de los encuestados señala que gestionan a nivel de aula la coenseñanza. Con menor aprobación, se observa el trabajo respecto a los ajustes metodológicos que se requieran según las necesidades de los estudiantes, de forma conjunta con el coenseñante.

En la dimensión IV - reflexión y promoción- (figura 5), los participantes poseen una valoración positiva de la estrategia de coenseñanza, por lo que es percibida como oportunidad de desarrollo profesional. De igual forma, un $60 \%$ asegura participar 
activamente en la formación de colegas en materia de coenseñanza. Llama la atención que solo un $20 \%$ considera que podría argumentar sobre los resultados de la coenseñanza en el aprendizaje de los estudiantes.

\section{Figura 4}

Dimensión III: ejecución de la coenseñanza

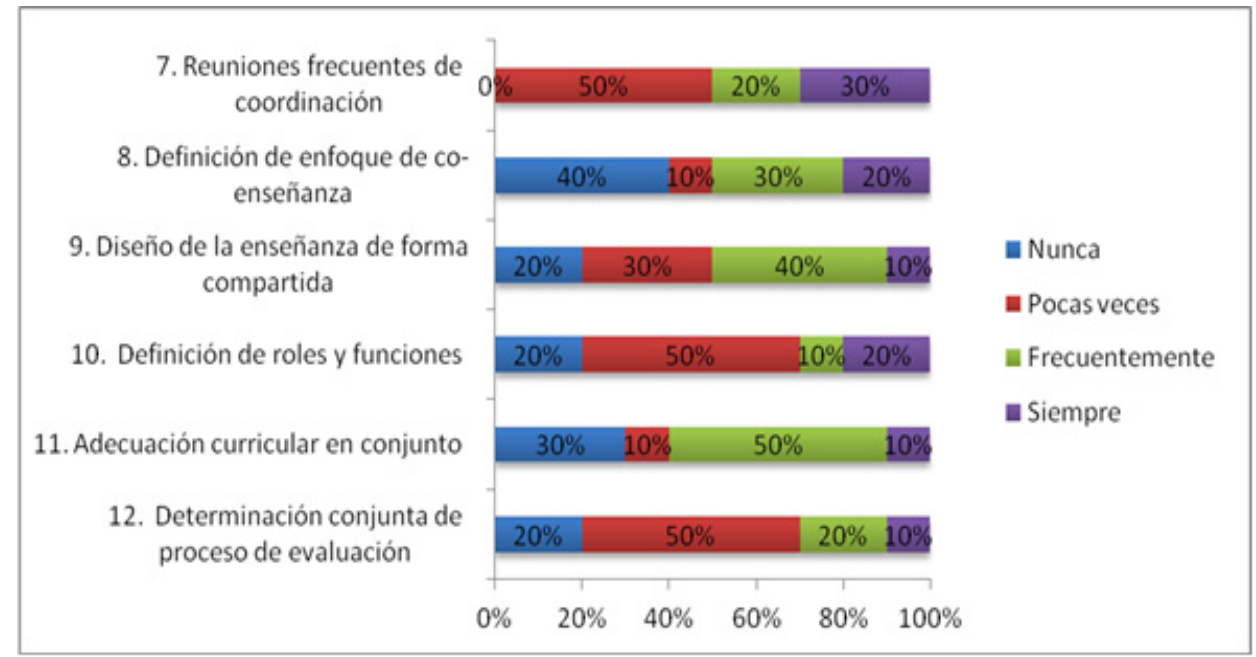

Figura 5

Dimensión IV: reflexión y promoción

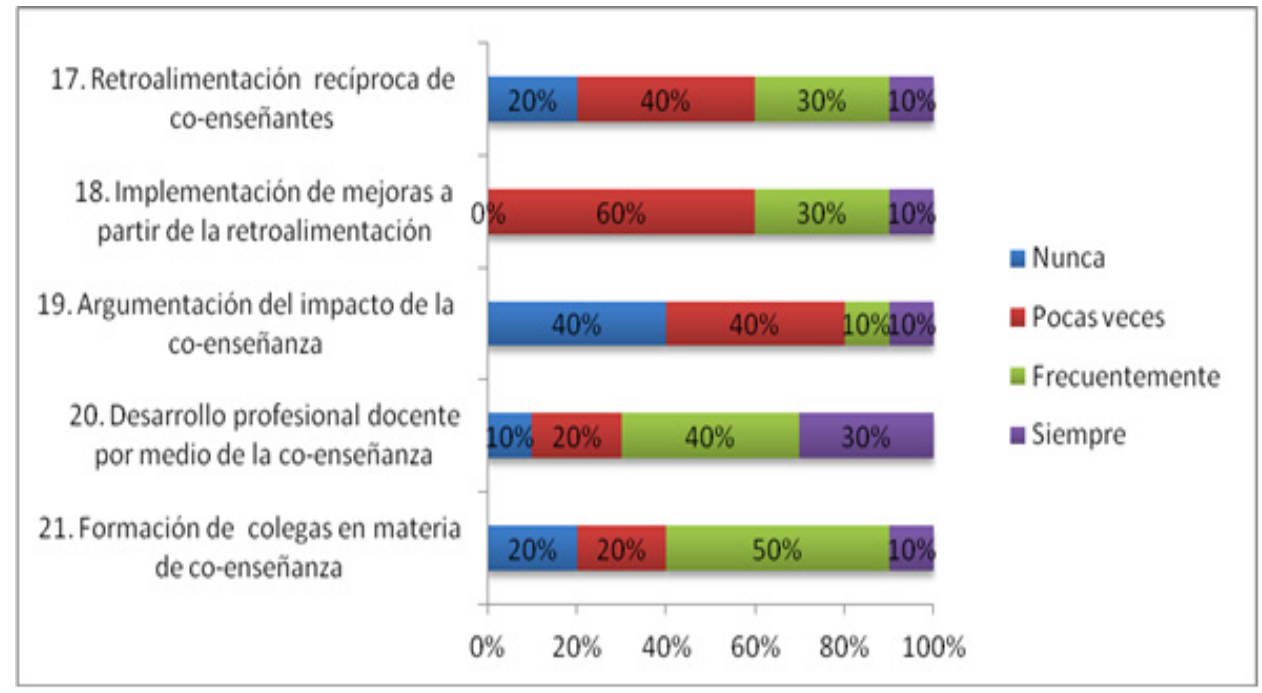

El análisis general de las cuatro dimensiones del estudio (figura 6) reveló que la dimensión cultura de colaboración adquiere el mayor porcentaje de aprobación por los encuestados, con un $77 \%$. Esta última se relaciona con la percepción de los docentes acerca de la estrategia de coenseñanza. Por otra parte, respecto a la dimensión ejecución, un $57 \%$ de los participantes aprueba la implementación en el aula de la coenseñanza. La dimensión promoción y reflexión alcanza resultados más bajos. Los docentes expresan que no logran argumentar resultados convincentes respecto de la relación entre evaluación y mejora de la implementación de la estrategia. Percepción similar expresan sobre la dimensión planificación, que aborda la preparación de la enseñanza y sus implicaciones didácticas. 


\section{Figura 6}

Análisis general de las dimensiones de evaluación de las prácticas de coenseñanza

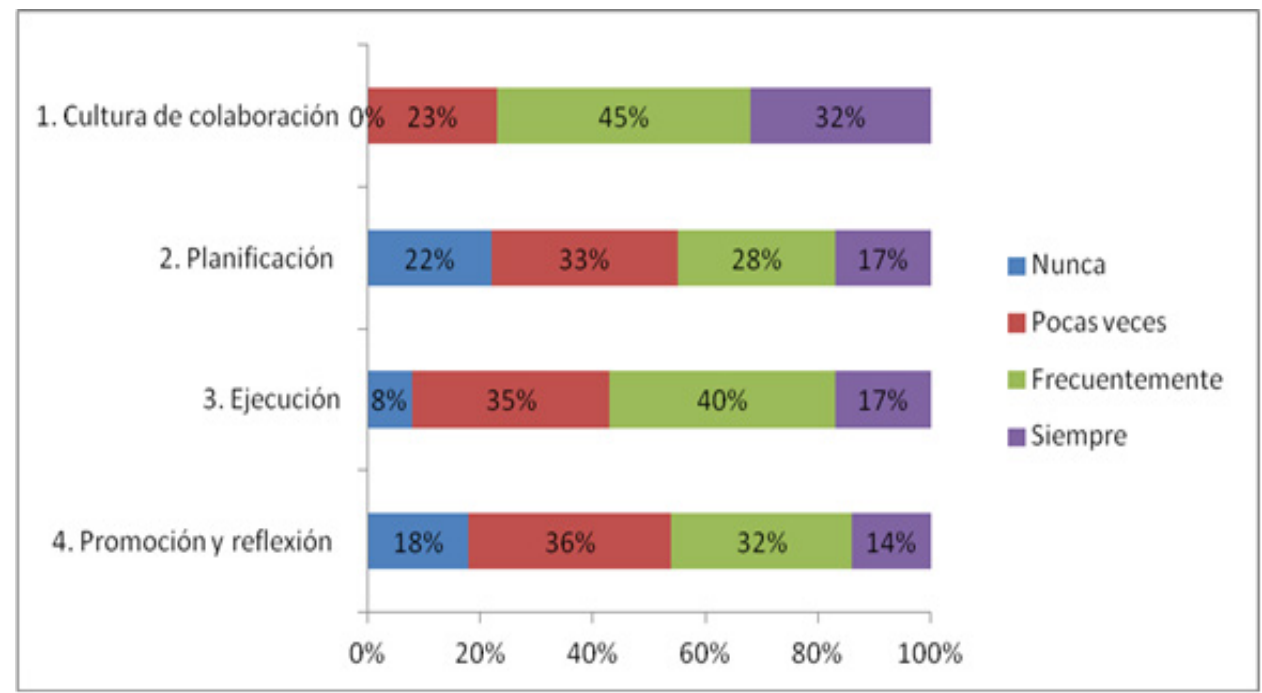

Una vez aplicada la entrevista, se representaron las percepciones de los docentes del primer grupo focal. La interpretación cualitativa de los resultados y el registro de las observaciones de los participantes en una etapa inicial se representa en la figura 7, que se obtiene mediante el software Atlas.ti (Murawski \& Swanson, 2001).

\section{Figura 7}

Análisis cualitativo de las percepciones del primer grupo focal

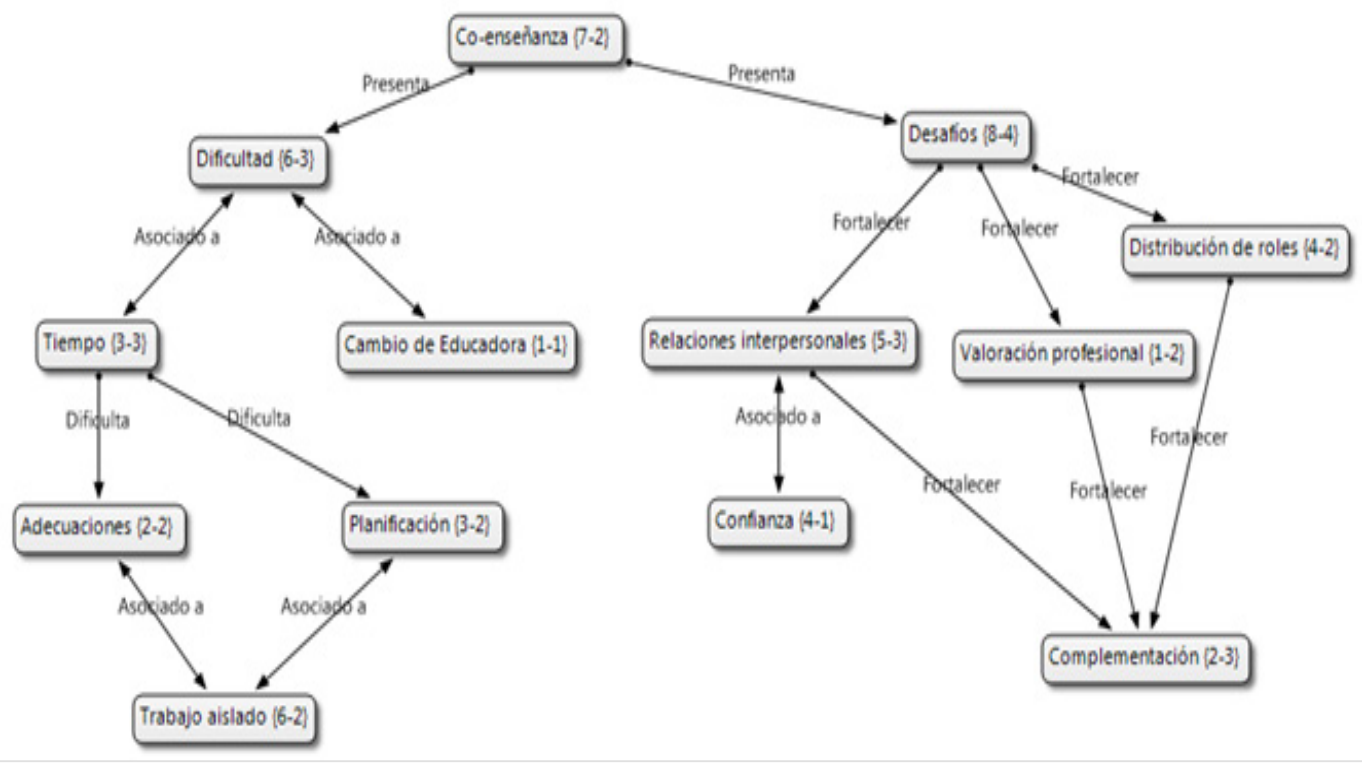

En el grupo focal, los docentes señalan que, para el desarrollo efectivo de la coenseñanza, existen dificultades, con énfasis en el tiempo de planificación de la enseñanza, en particular, porque las horas destinadas a las reuniones de los coenseñantes tienden a ser reorientadas a otras actividades o funciones, por ejemplo: atención de apoderados, cubrir cursos con docentes ausentes y reuniones. La situación planteada genera que, a nivel de aula, se 
desarrolle un trabajo aislado entre los docentes de aula común y la educadora diferencial. Esta última es un apoyo pasivo que se encarga de realizar las adaptaciones curriculares según las planificaciones diseñadas por la docente. Otra dificultad identificada, con menor énfasis, es el cambio de educadora diferencial durante el año escolar.

Entre los desafíos para mejorar la coenseñanza, se menciona que es necesario fortalecer las relaciones interpersonales entre docentes de aula regular y la educadora diferencial. Este punto se presenta con mayor prevalencia y se relaciona directamente con la confianza que debe existir entre los equipos de coenseñanza. También se presenta el desafío de distribuir los roles en el aula desde la preparación de la enseñanza.

A partir de estos resultados, se aplicaron talleres - no abordados en este artículo- con los docentes. En un segundo momento, se procede a la aplicación de las entrevistas, de las que se obtienen nuevas evidencias.

En la dimensión I - cultura de colaboración- (figura 8), los encuestados señalan que tienen una valoración positiva del proceso de coenseñanza y la consideran como un factor clave para desarrollar una clase efectiva. Por otra parte, un $70 \%$ declara que siempre se resuelven positivamente los conflictos pedagógicos que surgen entre coenseñantes. Un $60 \%$ menciona que comparten ideas comunes respecto del proceso de enseñanza. Asimismo, dicen que logran desarrollar una visión participativa del proceso, logran modelar la colaboración y el trabajo en equipo para los estudiantes y comparten la responsabilidad de la enseñanza desde la preparación hasta la reflexión y su evaluación.

\section{Figura 8}

Dimensión I: cultura de colaboración

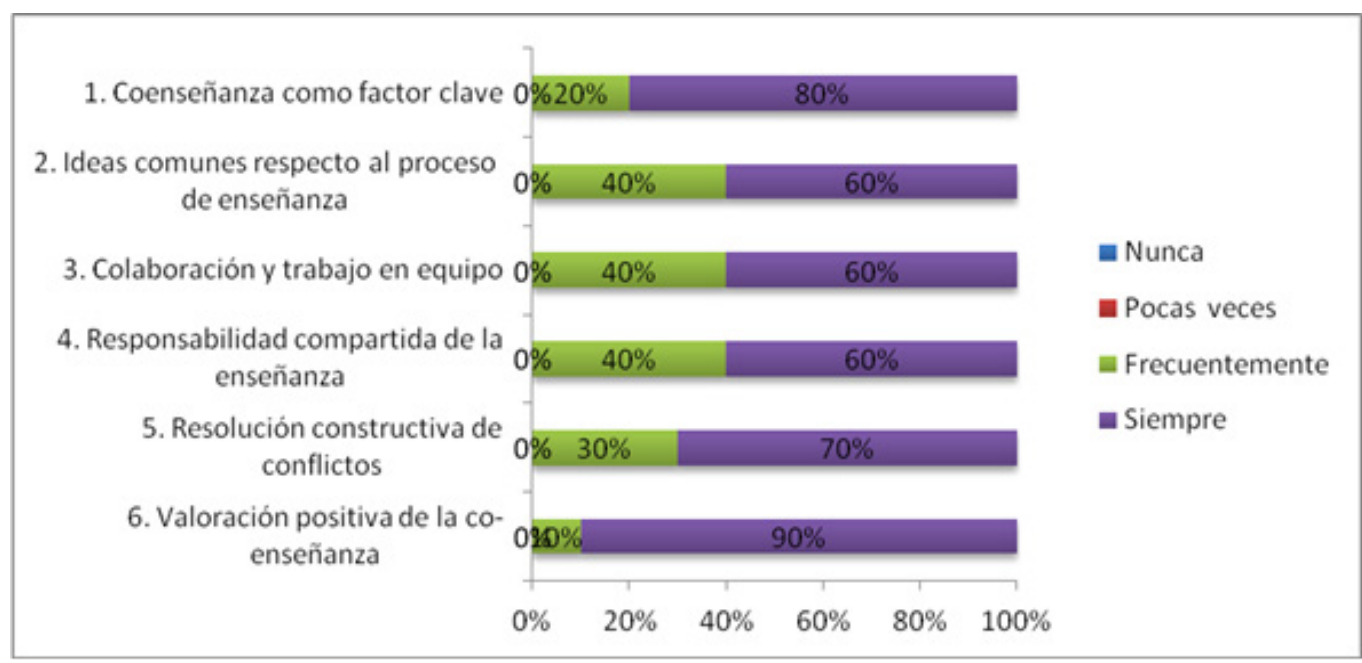

En relación con la dimensión II —planificación- (figura 9), los encuestados señalan que las adecuaciones curriculares se realizan en conjunto. El 90 \% valoró como positiva la definición de roles y funciones en el aula y la definición del enfoque de coenseñanza a utilizar.

La percepción de los docentes sobre la dimensión III -ejecución- (figura 10), en general es positiva, pues un $80 \%$ de los encuestados señala que siempre utilizan varios enfoques de coenseñanza. Mencionan que un $60 \%$ de ellos siempre implementa los 
enfoques acordados en la etapa de preparación de la enseñanza. Un $40 \%$ señala que esto ocurre frecuentemente. Además, la mitad de los encuestados destaca que siempre se realizan los ajustes metodológicos según las necesidades de los estudiantes. Esto demuestra que no existe una visión compartida sobre la gestión del clima de aula.

\section{Figura 9}

Dimensión II: planificación de la coenseñanza

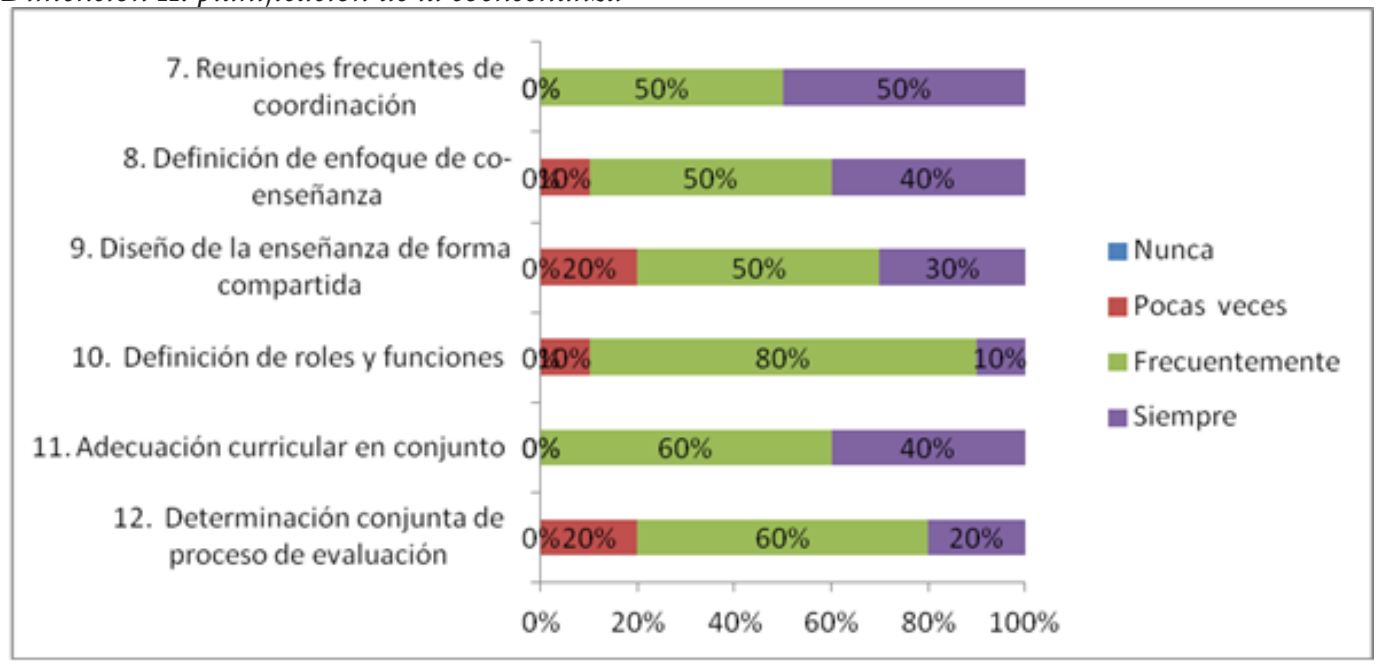

Figura 10

Dimensión III: ejecución de la coenseñanza

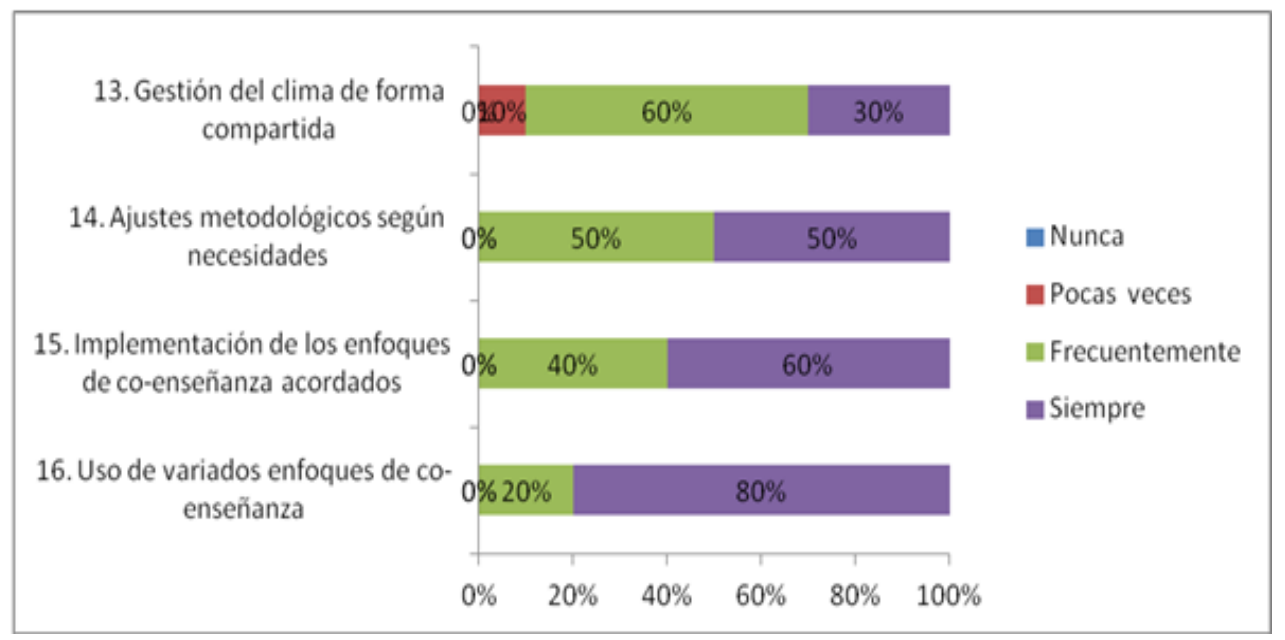

En relación con la dimensión IV - reflexión y promoción - (figura 11), en general, se evidencia una valoración positiva, por cuanto aumenta la aprobación del empleo de la coenseñanza como estrategia de trabajo entre profesionales. Un 80 \% está de acuerdo en su totalidad con esta afirmación. En esta dimensión se aprecia la existencia de una valoración positiva acerca de la retroalimentación entre coenseñantes mediante su desempeño y la implementación de las mejoras.

Como se observa en la figura 12, la dimensión cultura de colaboración es la que posee mayor porcentaje de aprobación y valoración positiva respecto de las concepciones y visión de la coenseñanza. Le sigue en resultados similares la dimensión ejecución de la 
coenseñanza, en la que los docentes declaran que implementan efectivamente los acuerdos del diseño de la enseñanza de forma compartida en el aula. Con menor porcentaje de aprobación, pero con similar comportamiento en la percepción positiva de los docentes, se encuentra la dimensión reflexión y promoción, que se relaciona con la evaluación y mejora de la implementación de la estrategia. Por último, la dimensión planificación posee un $90 \%$ de valoración positiva sobre el diseño de la enseñanza de forma compartida.

\section{Figura 11}

Dimensión IV: reflexión y promoción

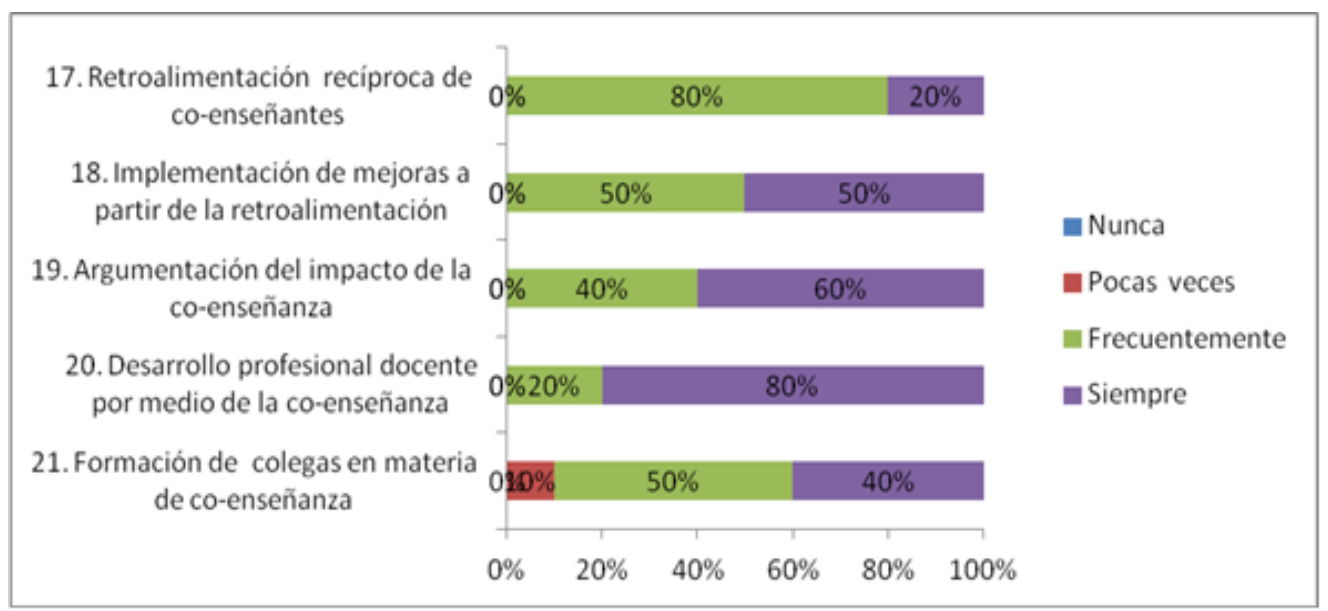

\section{Figura 12}

Análisis general de las dimensiones

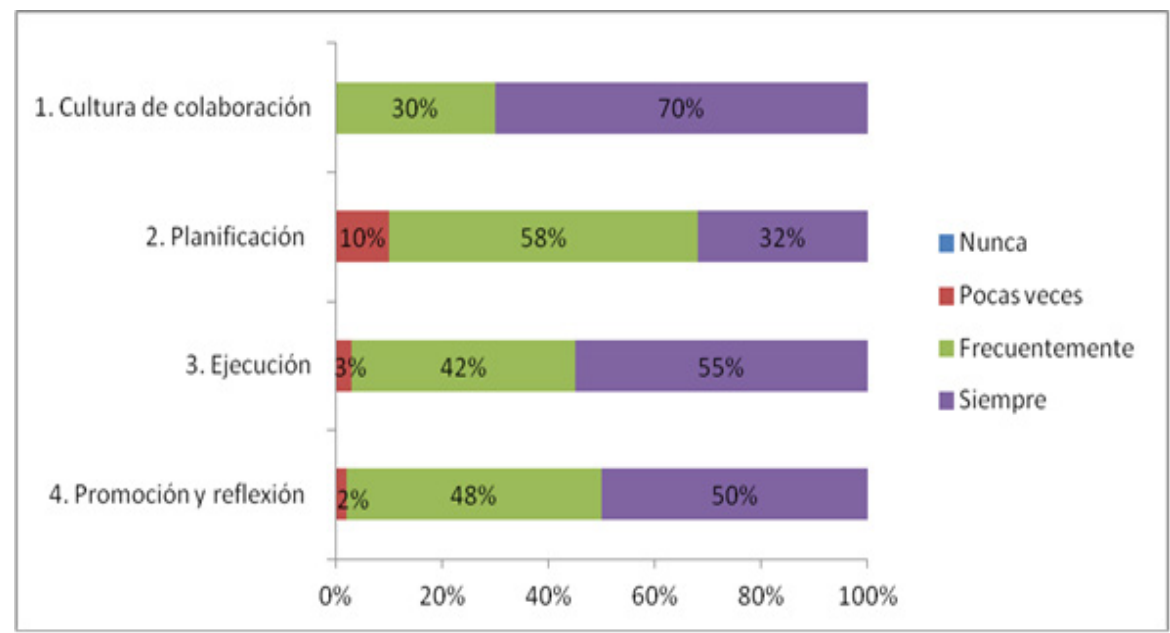

Según los antecedentes obtenidos del segundo grupo focal (figura 13), la coenseñanza ha desarrollado variadas mejoras que permiten contribuir al desarrollo profesional docente y al aprendizaje de los estudiantes.

También en el grupo focal se consensuó que las mejoras logradas se relacionan con la gestión de instancias colaborativas entre los profesionales del equipo del PIE y los docentes de aula. Esto último influyó de forma favorable en la actitud y disposición de los docentes, asunto que en el primer grupo focal fue objeto de crítica, puesto que los horarios de 
planificación no podían ejecutarse debido a situaciones emergentes que ocurren en horarios de clases.

\section{Figura 13}

Análisis de datos cualitativos, segundo grupo focal

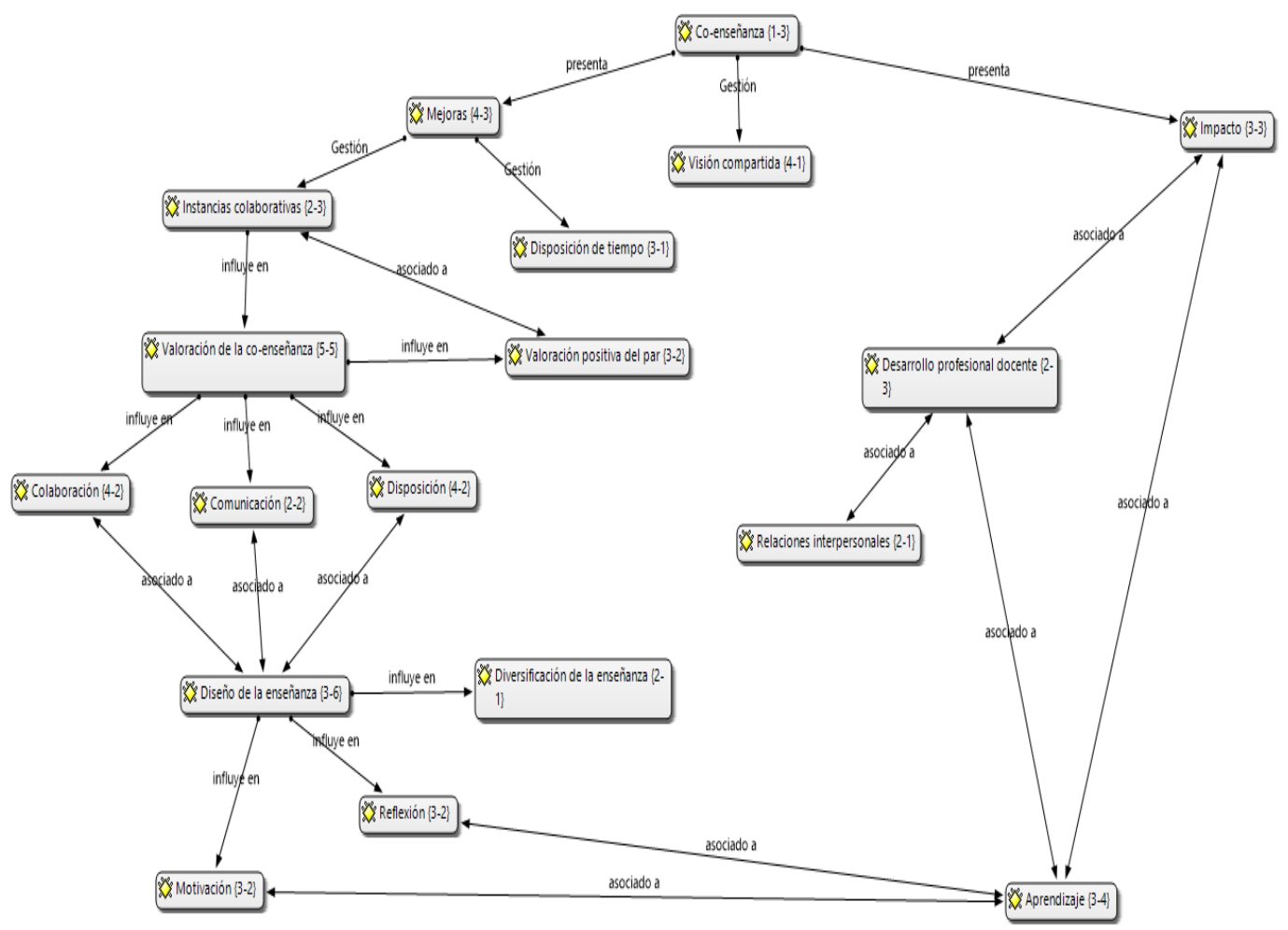

La dimensión planificación y los ajustes al respecto contribuyeron a: el establecimiento de instancias colaborativas entre profesionales, la valoración positiva de la coenseñanza, la gestión de diversos talleres y el establecimiento de jornadas de reflexión y aprendizaje que les han permitido identificar los beneficios de la coenseñanza. Se genera así una visión compartida de la coenseñanza, que comenzó a modificar las actitudes y comportamientos profesionales en materia de comunicación y la colaboración entre docentes.

La aplicación del instrumento ajustado y el grupo focal permitieron comprender la realidad del establecimiento en diferentes aspectos. Se creó el plan de mejoramiento continuo, en conjunto con los docentes, para fortalecer los aspectos que estaban más debilitados. Luego de su implementación, se establecieron las siguientes percepciones de los docentes:

- El trabajo colaborativo entre pares profesionales favorece el desarrollo profesional docente, el compromiso y la motivación.

- La motivación de los docentes trae consigo mayores expectativas sobre su trabajo, mejoras en sus prácticas y mayor reflexión.

- Los docentes de aula y las educadoras diferenciales del equipo del PIE fortalecen su trabajo profesional al gestionar instancias colaborativas de coenseñanza, reflexivas y evaluativas. 
- La efectiva gestión de mejora de las prácticas de coenseñanza trae consigo mayores oportunidades de aprendizaje para los estudiantes.

- La coenseñanza fortalece el liderazgo pedagógico y la gestión del currículum.

Los resultados obtenidos evidencian que la coenseñanza toma mayor sentido en las condiciones actuales, en las que se percibe como una herramienta para el trabajo colaborativo entre los docentes de aula y educadoras diferenciales. Contribuye a favorecer las relaciones interpersonales, la visión compartida de los problemas de la práctica pedagógica y fortalece el desarrollo profesional docente.

\section{Discusión}

Los orígenes de la coenseñanza se remontan a Estados Unidos, durante los años 50, ante los cuestionamientos acerca de la estructura tradicional, eficacia y eficiencia del sistema educativo. En los años 60 se comenzó a cuestionar la eficacia de la educación especial separada de la regular, lo que condujo a que padres de hijos con discapacidad desafiaran las barreras que debían enfrentar en el proceso educativo.

En los años 70, se da inicio a una incipiente legislación destinada a remediar las desigualdades educativas, que dio lugar a los primeros experimentos de colaboración entre docentes regulares y de educación especial. A finales de los años 80 , con la creciente masificación de los principios de inclusión, surgió el concepto de coenseñanza -en inglés, coteaching - como complemento del término enseñanza cooperativa - en inglés, cooperative teaching-(Murawski \& Swanson, 2001). En la literatura consultada, se define la coenseñanza como la unidad de acción entre dos o más profesionales con la responsabilidad común de enseñar a un grupo de estudiantes, al generar ayudas y apoyos de forma colaborativa, y en consecuencia con las necesidades educativas de los estudiantes (Altstaedter et al., 2016).

Desde sus inicios, la coenseñanza se llevó a cabo por un educador tradicional con manejo dela didáctica, del currículum y de la evaluación, junto a un educador diferencial con conocimientos respecto a las NEE. Ambos profesionales, con diferentes experiencias, brindaban respuestas más amplias frente a la necesaria formación y actualización para atender las necesidades educativas de los estudiantes. De esta forma, la intención de la coenseñanza desde sus orígenes fue hacer posible que los estudiantes, con o sin discapacidad —o con NEE- pudieran acceder al currículum general, mediados por profesionales competentes cuyas experiencias de aprendizaje se ajustaran a sus necesidades (Godoy, 2015; Mellado et al., 2017).

En Chile, el fomento de la coenseñanza tiene como contexto sociohistórico esencial la creación en los años 90 de los equipos del PIE. Además, la masificación del concepto de inclusión en Chile sentó las bases para la institucionalización en el año 2007 de la Ley 20.201 (MinEduc, 2007). Esta generó cambios en la subvención de educación especial e integró a los niños con diagnóstico de NEE de carácter transitorio y permanente. De esta ley se desprende el Decreto 170/2009 (MinEduc, 2009b), que actualmente rige el desempeño profesional de los equipos PIE. La implementación de ambas normativas en los establecimientos educativos sentó las bases jurídicas y pedagógicas para generar las Orientaciones Técnicas para PIE, que abordan el trabajo colaborativo y la coenseñanza (MinEduc, 2013b). No obstante, su desarrollo pleno en las prácticas y percepciones de la comunidad educativa requieren de un cambio y una mejora en la formación de los profesionales implicados en 
su materialización. Así, surge la necesidad de evaluar las prácticas de coenseñanza, dado su valor transformador, al retroalimentar y perfeccionar la educación de los sujetos con NEE o no incluidos en el aula regular.

Por otra parte, la evidencia científica encontrada en las publicaciones denota las potencialidades de la coenseñanza (Friend et al., 2010; Guisso \& Gesser, 2019; MinEduc, 2013b; Lagos, 2019; Rodríguez, 2014; Villa et al., 2008). Además, los argumentos enunciados justifican la pertinencia de la evaluación de la coenseñanza, de modo que los resultados conduzcan al fomento de prácticas colaborativas y se instruya a los docentes en relación con los resultados de la coenseñanza en el aula mediante la reflexión y la retroalimentación. Todo esto sienta las bases para perfeccionar la gestión de calidad de la educación mediante la transferencia de acciones de mejora continua en los establecimientos.

\section{Conclusiones}

La investigación se centró en la evaluación de las prácticas de coenseñanza. Los resultados de las entrevistas y los grupos focales permitieron obtener evidencias acerca del nivel de efectividad de las estrategias de coenseñanza según la percepción de los docentes participantes en el estudio, lo que confirmó la conjetura inicial formulada tras el estudio de la Fundación Chile (2013), por lo que afirmamos que, en el establecimiento objeto de estudio, las orientaciones actuales del MinEduc respecto a la coenseñanza no son del dominio y la comprensión plena por los docentes, lo que se comprobó en la percepción respecto de su implementación, reflexión y promoción. Asimismo, el hecho de contar con profesionales en el equipo del PIE como potencialidad favorece el fomento de prácticas de evaluación basadas en la estrategia de coenseñanza, como establece el MinEduc.

La aplicación del estudio, con auxilio de la adaptación del instrumento Are we really coteachers?" (Villa et al., 2008) para las entrevistas y el grupo focal, dio los primeros indicios sobre la percepción de los profesionales en torno al nivel de efectividad de la coenseñanza. Una vez aplicados los talleres - no presentados en este artículo- con los docentes, se efectúo el segundo grupo focal. En este se obtuvieron nuevas evidencias sobre: la percepción de fortalezas y necesidades en materia de coenseñanza en los docentes de aula y educadores diferenciales del establecimiento. Se creó un plan de acción en conjunto con los docentes y se acordaron acciones para implementar en el plan de mejoramiento de la escuela.

Las entrevistas y el registro de las percepciones en los grupos focales condicionaron la obtención de evidencias sobre el empleo de la estrategia de coenseñanza, dada su influencia en la mejora del desarrollo profesional docente. Aun así, es necesario profundizar en variables tales como: (1) la evaluación comprensiva de la influencia de la coenseñanza en el aprendizaje de los estudiantes; (2) la instauración sostenible de instancias reflexivas y de retroalimentación de las estrategias de coenseñanza; y (3) la ejecución de programas de capacitación y su evaluación comprensiva a partir de las proyecciones en la construcción de buenas prácticas pedagógicas por el cuerpo docente.

\section{Referencias}

Altstaedter, L., Smith, J., \& Fogarty, E. (2016). Co-teaching: towards a new model for teacher preparation in foreign language teacher education. Hispania, 99(4), 635-649. https:// www.jstor.org/stable/44114649 
Arriagada, C., \& Calzadilla-Pérez, O. (2018). Percepción de las bases curriculares de la educación básica multigrado en La Araucanía, Chile. Praxis \& Saber, 9(20), 75-95. https://doi.org/10.19053/22160159.v9.n20.2018.8296

Friend, M., Cook, L., Hurley-Chamberlain, D., \& Shamberger, C. (2010). Co-Teaching: An Illustration of the Complexity of Collaboration in Special Education. Journal of Educational and Psychological Consultation, 20(1), 9-27. https://doi.org/10.1080/10474410903535380

Fundación Chile. (2013). Análisis de la implementación de los programas de integración escolar (PIE) en establecimientos que han incorporado estudiantes con necesidades educativas especiales transitorias. http://portales.mineduc.cl/usuarios/edu.especial/ doc/201402101720120.ResumenEstudi oImplementacionPIE2013.pdf

Godoy, X. (2015). Complejidades del proceso de inclusión educativa para estudiantes con discapacidad en los centros educativos de Chile. Revista Integra Educativa, 8(2), 165-175. http://www.scielo.org.bo/scielo.php?script=sci_arttext\&pid=S199740432015000200010\&lng=es\&nrm=iso\&tlng=es

González, J. (2010). Pasado, presente y futuro de la atención a las necesidades educativas especiales: hacia una educación inclusiva. Perspectiva Educacional, Formación de Profesores, 49(1), 62-89. https://www.redalyc.org/pdf/3333/333327288003.pdf

Guirado, V., García, C., Navarro, X., \& Martín, D. (2017). Bases teórico-metodológicas para la atención a la diversidad y la inclusión educativa. Revista Universidad y Sociedad, 9(3), 82-88. http://scielo.sld.cu/scielo.php?script=sci_arttext\&pid=S2218$36202017000300012 \& \operatorname{lng}=$ es\&tlng=es.

Guisso, L., \& Gesser, M. (2019). Docência e processos de escolarização: desafios nos anos iniciais do ensino fundamental. Psicologia: Ciência e Profissão, 39, e186536. https://doi. org/10.1590/1982-3703003186536

Hernández, R., Fernández, C., \& Baptista, M. (2006). Metodología de la investigación (4 ed.). McGraw Hill Educación.

Iglesias, S. (2018). Los estudiantes con necesidades educativas especiales. Recursos y apoyos para su atención educativa. Revista Electrónica en Educación y Pedagogía, 2(2), 51-66. https://revedupe.unicesmag.edu.co/index.php/EDUPE/article/view/11

Lagos, O. (2019). Diseño universal para el aprendizaje: una experiencia innovadora en el aula matemática de octavo año básico. Revista de Estudios y Experiencias en Educación, 18(36), 257-267. https://dx.doi.org/10.21703/rexe.20191836lagos3

Marsiglia-Fuentes, R., Llamas-Chávez, J., \& Torregroza-Fuentes, E. (2020). Las estrategias de enseñanza y los estilos de aprendizaje una aproximación al caso de la licenciatura en educación de la Universidad de Cartagena (Colombia). Formación Universitaria, 13(1), 27-34. https://dx.doi.org/10.4067/S0718-50062020000100027

Mella, O. (2010). Grupos Focales. Técnica de investigación cualitativa. http://biblioteca. uahurtado.cl/ujah/856/txtcompleto/txt105091.pdf

Mellado, M., Chaucono, J., Hueche, M., \& Aravena, O. (2017). Percepciones sobrela educación inclusiva del profesorado de una escuela con Programa de Integración Escolar. Revista 
Educación, 41(1), 119-132. https://dx.doi.org/10.15517/revedu.v41i1.21597

Ministerio de Educación de Chile. (30 de septiembre, 1990). Decreto Supremo Exento $N^{o}$ 490/90. Normas técnicas y administrativas para integrar alumnos con discapacidad en establecimientos comunes (Jardines, Escuelas Básicas y/o Liceos). Biblioteca del Congreso Nacional de Chile. https://www.leychile.cl/ Navegar?idNorma $=13743 \&$ buscar $=\mathrm{ds}+490+1990$

Ministerio de Educación de Chile. (3 de julio, 2007). Ley 20.201. Modifica el DFL $N^{o}$ 2, de 1998, de educación, sobre subvenciones a establecimientos educacionales y otros cuerpos legales. Biblioteca del Congreso Nacional de Chile. https://www.leychile.cl/ Navegar?idNorma $=263059$

Ministerio de Educación de Chile. (12 de septiembre, 2009a,). Ley No. 20.536. Ley General de Educación. Biblioteca del Congreso Nacional de Chile. https://www.leychile.cl/ Navegar?idLey $=20370$

Ministerio de Educación de Chile. (21 de abril, 2009b). Decreto $N^{\circ}$ 170/2009. Normas para determinar los alumnos con necesidades educativas especiales que serán beneficiarios de la subvención para educación especial. Biblioteca del Congreso Nacional de Chile. https:// especial.mineduc.cl/normativa/decretos-e-instructivos/

Ministerio de Educación de Chile. (2013a). Antecedentes Generales PIE. Unidad de Educación Especial. https://www.ayudamineduc.cl/ficha/antecedentes-generales-pie-5

Ministerio de Educación de Chile. (2013b). Orientaciones Técnicas para Programas de Integración Escolar. Unidad de Educación Especial. https://especial.mineduc.cl/wpcontent/uploads/sites/31/2016/09/Orientaciones-PIE-2013-3.pdf

Ministerio de Educación de Chile. (2016). Manual de apoyo a sostenedores y establecimientos educacionales, para la implementación del Programa de Integración Escolar (PIE), en el marco de la Inclusión. Coordinación de Programas de Integración Escolar y Unidad de Educación Especial de la División de Educación General. https:// especial.mineduc.cl/wp-content/uploads/sites/31/2017/12/Manual-PIE.pdf

Ministerio de Educación de Chile. (2 de diciembre, 2019). Propuestas de mesa técnica por las Necesidades Educativas Especiales. Unidad de Educación Especial, División de Educación General. https://especial.mineduc.cl/propuestas-de-mesa-tecnica-por-necesidadeseducativas-especiales/

Molina, Y. (2015). Necesidades educativas especiales, elementos para una propuesta de inclusión educativa a través de la investigación acción participativa: el caso de la Escuela México. Estudios pedagógicos, 41(especial), 147-167. https://dialnet.unirioja.es/servlet/ articulo? codigo $=3678789$

Moran, M., Vera, L., \& Morán, M. (2017). Los trastornos del lenguaje y las necesidades educativas especiales: consideraciones para la atención en la escuela. Revista Universidad y Sociedad, 9(3), 191-197. http://scielo.sld.cu/scielo.php?script=sci_arttext\&pid=S221836202017000300030\&lng=es\&tlng=pt

Murawski, W. (2008). Five keys to co-teaching in inclusive classrooms. School Administrator, 
65(8), 27. https://www.csun.edu/sites/default/files/Five-Keys-Co-Teaching-InclusiveClassroom.pdf

Murawski, W., \& Swanson, H. (2001). A meta-analysis of co-teaching research. Where are the data? Remedial and Special Education, 22(5), 258-267. https://journals.sagepub.com/ doi/abs/10.1177/074193250102200501

OCDE. (3 de junio, 2004). Revisión de políticas nacionales de educación. https:// www.oecd-ilibrary.org/education/revision-de-politicas-nacionales-de-educacionchile_9789264021020-es

ONU. (1993). Normas uniformes, sobre la igualdad de oportunidades para las personas con discapacidad. https://www.who.int/disabilities/policies/standard_rules/es/

Rodríguez, F. (2014). La co-enseñanza, una estrategia para el mejoramiento educativo y la inclusión. Revista Latinoamericana de Educación Inclusiva, 8(2), 219-233. https:// dialnet.unirioja.es/descarga/articulo/4994333.pdf

Soto, N. (2016). La atención educativa de niños, niñas y jóvenes considerados con necesidades educativas especiales: una mirada desde la integración y desde la inclusión. https:// repository.cinde.org.co/handle/20.500.11907/470

Suárez, G. (2016). Co-enseñanza: concepciones y prácticas en profesores de una facultad de educación en Perú. Revista Electrónica de Investigación Educativa, 18(1), 166-182. http:// www.scielo.org.mx/scielo.php?script=sci_arttext\&pid=S1607-40412016000100012\&ln $\mathrm{g}=\mathrm{es} \& \mathrm{t} \operatorname{lng}=\mathrm{es}$.

Unesco. (1990). Conferencia Mundial de Educación para Todos. https://unesdoc.unesco.org/ ark:/48223/pf0000184556

Unesco. (1994). Declaración de Salamanca y Marco de Acción Sobre Necesidades Educativas Especiales. Conferencia Mundial sobre Necesidades Educativas Especiales: Acceso y Calidad. https://unesdoc.unesco.org/search/N-EXPLORE-8f374fea-fb78-4788-94cd8dd764d505e4

Unesco. (2000). Marco de Acción del Foro Mundial de Educación para Todos. https://unesdoc. unesco.org/ark:/48223/pf0000121147_spa

Urbina, C., Basualto, P., Durán, C., \& Miranda, P. (2017). Prácticas de co-docencia: el caso de una dupla en el marco del Programa de Integración Escolar en Chile. Estudios pedagógicos, 43(2), 355-374. https://dx.doi.org/10.4067/S0718-07052017000200019

Vance, A. (2001). Teacher's beliefs about co-teaching. Remedial and Special Education, 22(4), 245-255. https://journals.sagepub.com/doi/abs/10.1177/074193250102200408

Villa, R., Thousand, J., \& Nevin, A. (2008). Una guía para la co-enseñanza: consejos prácticos para facilitar el aprendizaje de los estudiantes (vol. 2). Corwin Press. 